\title{
The Challenges of Pertussis Outbreaks in Healthcare Facilities: Is There A Light at the End of the Tunnel?
}

\author{
Kathryn M. Edwards, MD; Thomas R. Talbot, MD, MPH
}

Although classically recognized as a disease of infants and children, the reported incidence of Bordetella pertussis infection in adolescents and adults has increased at a remarkable rate over the past decade. During 2004, more than 25,000 cases of pertussis were reported to the Centers for Disease Control and Prevention (CDC), the highest total since 1959; of these, $34 \%$ were in individuals aged $11-18$ years and $29 \%$ were in adults aged 19 years and older. ${ }^{1}$ This increase in pertussis in adolescents and adults is likely due to several factors, including waning protection from childhood vaccination and natural infection, an increased appreciation of disease in this age group, and the improved ability of clinicians to diagnose pertussis through the use of serologic methods. Whether this increase in pertussis incidence represents a true increase in disease or just reflects an improved recognition of disease remains unclear. However, what is clear is, first, that rates of pertussis are also increasing in infants, and second, that adolescents and adults serve as a source of contagion for infants and young children, who suffer the greatest morbidity, and even mortality, from pertussis. Despite high rates of routine pertussis vaccination among children, ${ }^{2}$ rates of pertussis disease continue to $\operatorname{climb}$, and it is becoming a major public health problem.

Healthcare-associated outbreaks of pertussis also have been increasingly recognized and reported from a diverse range of healthcare facilities, such as residential homes for mentally or physically impaired persons, pediatric wards and nurseries, emergency departments, and hematology-oncology care units. ${ }^{3-11}$ Such outbreaks are often the result of under-recognition of pertussis and subsequent failure to isolate patients with suspected infection, as well as the increasing incidence of pertussis among adults and adolescents. Healthcare workers (HCWs) are at increased risk for acquiring $B$. pertussis infection; one study estimates that there is a 1.7-fold increased risk for $\mathrm{HCWs}$, compared with the general population. ${ }^{12} \mathrm{~A}$ serologic analysis of samples from emergency department HCWs at Vanderbilt University Medical Center revealed annual incidence rates of 1.3 infections per 100 persons among emergency department resident physicians and 3.6 infections per 100 persons among emergency department nursing and patient care staff. ${ }^{13}$ This increased risk is likely the result of regular contact with infected patients and waning protection from childhood vaccination or natural $B$. pertussis infection. Infected HCWs can then serve as vectors of infection to other susceptible contacts, including patients, other employees, and even their own children. ${ }^{10}$

Nosocomial exposure to $B$. pertussis results in substantial disruptions and costs to the healthcare system, to HCWs, and to individual contacts. Costs include, but are not limited to, person-hours spent by infection control and occupational health staff investigating the contacts of an index patient and of exposed persons, laboratory evaluation of symptomatic contacts, antimicrobial prophylaxis for exposed individuals, efforts to alert communities of pertussis outbreaks, uncompensated medical costs borne by contacts, and loss of productivity and wages from HCWs who are ill and/or furloughed. As would be expected, such costs are not trivial. For example, an evaluation of employees and patients exposed to 3 HCWs with documented pertussis in a French hospital cost more than $€ 46,000$, with $42 \%$ of that figure the result of productivity losses. ${ }^{14}$ In another healthcare-associated outbreak of pertussis in $17 \mathrm{HCWs}$ exposed to an infected infant, the evaluation of $307 \mathrm{HCW}$ contacts resulted in more than $\$ 81,000$ in total measured costs. ${ }^{15}$

In this issue of the journal, several articles complement our growing knowledge about nosocomial pertussis outbreaks. Three of them highlight the role of adults in transmission of pertussis to infants, the deficiencies in the health care setting that allow $B$. pertussis transmission to occur readily, and the challenges in managing nosocomial exposures to pertussis. Perhaps the most distressing account is the article by Bryant et al., ${ }^{16}$ which describes a severe $B$. pertussis infection in a premature infant that was traced back to an ill HCW. As noted by the authors, "despite paroxysms, whoop, posttussive emesis, a spontaneous pneumothorax, and multiple visits for medical care, pertussis was not suspected [in the

From the Divisions of Infectious Diseases in the Department of Pediatrics (K.M.E.) and Department of Medicine (T.R.T.), Pediatric Clinical Research Office (K.M.E.), and Department of Preventive Medicine (T.R.T.), Vanderbilt University School of Medicine, Nashville, Tennessee.

Received April 14, 2006; accepted April 14, 2006; electronically published May 26, 2006.

Infect Control Hosp Epidemiol 2006; 27:537-540

(c) 2006 by The Society for Healthcare Epidemiology of America. All rights reserved. 0899-823X/2006/2706-0001\$15.00. 
HCW] until diagnosis of the infant case" (p. 543). In total, 79 patients and 72 HCWs received postexposure treatment. Although recent publications have highlighted that pertussis is responsible for $7 \%-32 \%$ of cough illnesses that last 1 week or longer in adolescents and adults, ${ }^{17-19}$ many clinicians fail to recognize the disease, and most have great difficulty with laboratory confirmation of the disease. B. pertussis culture has been widely available for many years, and polymerase chain reaction methods have recently been added to the diagnostic repertoire, but in many instances, by the time clinicians suspect pertussis in adults, neither of these studies will have yielded positive results. Although serologic testing will confirm the diagnosis in adults, it is not widely available to clinicians. The CDC and the US Food and Drug Administration have been collaborating to provide a serologic test that would be widely available for clinicians, but it is not yet operational. If the diagnosis of pertussis had been considered sooner for the symptomatic HCW described by Bryant et al., ${ }^{16}$ and the diagnosis confirmed by serologic testing, then the entire outbreak in the neonatal ward might have been averted.

Another striking yet all-too-common finding in the outbreak reported by Bryant et al. ${ }^{16}$ is the difficulty of tracing HCWs exposed to the index patient. Lack of documentation about HCWs who provided key direct patient care activities, such as bathing and feeding, led to an incomplete identification of exposed personnel. Such personnel, if not identified and offered appropriate postexposure prophylaxis, could have caused further disease transmission. Such problems with contact tracing are not unique to $B$. pertussis exposure, and they serve to emphasize the need to develop more detailed documentation tools to track contact between HCWs and patients.

The report by Bamberger et al. ${ }^{20}$ demonstrates the global nature of the pertussis problem; it describes 2 outbreaks of nosocomial pertussis at 2 different healthcare facilities in Israel. In both instances, the infected infants resided in adjacent beds, and the probable source of infection was a symptomatic parent of one of the infants. Failure to detect a history of cough in the parent and allowing contact between the parent and other infants in the ward facilitated the nosocomial acquisition of $B$. pertussis. The restriction of ill visitors in healthcare institutions is often highlighted in strategies to prevent nosocomial spread of respiratory viral infections, such as influenza and respiratory syncytial virus infection. However, questioning and restriction of visitors with a nonfebrile cough illness is often not a component of such policies. As illustrated by the outbreaks in Israel, it must be ensured that coughing visitors (regardless of whether they have a febrile illness) have limited access to patients in the healthcare setting, particularly in high-risk units such as pediatric intensive care units and newborn nurseries. In addition, visitor contact with other patients should be limited. As Bamberger et al. ${ }^{20}$ suggest, "nurseries should discourage parents from contact with infants other than their own" (p. 624).

Another key issue with regard to nosocomial pertussis is the use of antimicrobial postexposure prophylaxis. For many years, the traditional agent used in postexposure prophylaxis for HCWs exposed to pertussis has been erythromycin. An effective agent in preventing infection in exposed persons, ${ }^{21}$ the drug's major drawback is that its use is associated with a substantial occurence of adverse events, particularly nausea, emesis, and diarrhea. Although newer macrolides with a lower associated frequency of adverse effects have been developed (eg, azithromycin), they were long considered second-line agents for postexposure prophylaxis for pertussis in formal recommendations. ${ }^{23,24}$ Devasia and colleagues ${ }^{25}$ evaluated the tolerability of erythromycin and azithromycin postexposure prophylaxis during a community-wide pertussis outbreak in Tennessee. Only $57 \%$ of persons who were prescribed erythromycin for postexposure prophylaxis completed the antibiotic course, compared with $94 \%$ who were prescribed azithromycin. The medication's adverse effects (predominantly gastrointestinal) were cited as the reason for nonadherence to therapy by $33 \%$ of the erythromycin group, compared with only $4 \%$ of the azithromycin group. Before adoption of azithromycin as a first-line option for postexposure prophylaxis, the perceived risk of erythromycin-associated adverse events led to HCWs in our hospital to refuse the provided erythromycin postexposure prophylaxis in favor of azithromycin they paid for out of pocket.

Recently, the CDC released revised recommendations for treatment of and postexposure prophylaxis for pertussis in which any macrolide (erythromycin, azithromycin, or clarithromycin) is considered a first-line agent for postexposure prophylaxis in adults and children. ${ }^{21}$ For neonates younger than 1 month, azithromycin is the recommended agent for postexposure prophylaxis because of the association of erythromycin with infantile hypertrophic pyloric stenosis. The addition of azithromycin to the first-line agents for postexposure prophylaxis should improve adherence to postexposure prophylaxis, thus reducing secondary transmission of $B$. pertussis in healthcare facilities.

The outbreak of pertussis in Paris, France, described by Giugliani et al..$^{22}$ in this issue highlights additional challenges. The authors describe an outbreak of pertussis in an internal medicine ward and their attempt to deliver azithromycin prophylaxis to more than $200 \mathrm{HCWs}$. Even with the use of azithromycin, $33 \%$ of HCWs reported at least 1 adverse event, and $11 \%$ of the HCWs refused treatment. As shown in the article by Bryant et al., ${ }^{16}$ the refusal of prophylaxis by even a single $\mathrm{HCW}$ can set off a second wave of contagion and require additional rounds of prophylaxis. Another interesting finding in the study by Giugliani et $\mathrm{al}^{22}$ is that, although the adverse effects of erythromycin prophylaxis for pertussis are widely appreciated, the adverse effects associated with the "better tolerated" azithromycin still resulted in discontinuation of therapy by $8 \%$ of HCWs. The question of whether HCWs are more willing to tolerate adverse effects of medication for treatment of their own disease than for prevention of disease in others is merely speculative.

Taken together, these 3 informative studies could fill read- 
ers with concern about how we might control pertussis in the healthcare setting. However, the recent licensure, in 2005, of 2 acellular pertussis vaccines combined with tetanus and diphtheria toxoids (Tdap vaccines) for use as adolescent boosters (and, for one product, use as an adult booster) is cause for great optimism. ${ }^{1,26}$ Childhood vaccination against $B$. pertussis was first recommended in the 1940 s, and at that time pertussis vaccines consisted of diphtheria and tetanus toxoids combined with killed whole-cell $B$. pertussis organisms. These vaccines were associated with high rates of local and systemic adverse effects. ${ }^{19} \mathrm{~A}$ less reactogenic vaccine, which replaced the whole-cell pertussis vaccine with a more highly purified acellular $B$. pertussis component (Tdap), was licensed in 1991. With universal childhood pertussis vaccination, the incidence of pertussis in children has markedly declined. ${ }^{1}$ Tdap vaccines are also highly effective in adolescents and adults, as illustrated by the recently published Adult Pertussis Trial. ${ }^{27}$ In this randomized, clinical trial, which enrolled more than 2,700 healthy adults and adolescents, administration of an acellular pertussis vaccine was $92 \%$ efficacious in preventing pertussis confirmed by culture or serologic testing. ${ }^{27}$

In the past several years, 2 expert groups, the International Consensus Group on Pertussis Immunisation ${ }^{28}$ and the Global Pertussis Initiative, ${ }^{29}$ have both recommended universal adolescent booster pertussis vaccination combined with targeted vaccination of those adults most likely to have contact with infants, including parents and other close family members, HCWs, and daycare workers; universal vaccination of young adults; and routine use of Tdap vaccines rather than the adult tetanus and diphtheria toxoid vaccine for all adult booster vaccinations. In view of the increasingly recognized problem of healthcare-associated $B$. pertussis infection, the CDC's Advisory Committee on Immunization Practices targeted HCWs as a priority group for pertussis vaccination in 2006, primarily to reduce the risk of spread of B. pertussis within healthcare institutions. ${ }^{26}$

The use of Tdap vaccines for vaccination of HCWs heralds an exciting era in the prevention of nosocomial pertussis; however, questions remain regarding the impact and implementation of Tdap vaccination of HCWs. Does the use of the highly efficacious vaccine eliminate the need for any additional postexposure prophylaxis for vaccinated HCWs who have been exposed to pertussis? What is the duration of protection in a vaccinated cohort, and should booster vaccinations be given to those at high risk of exposure? If so, how frequently should such booster vaccinations occur? Universal vaccination of HCWs with Tdap vaccines may abolish the need for widespread postexposure prophylaxis and allow limited resources to be directed towards other preventive efforts. It has been estimated that vaccination of HCWs with Tdap vaccines would prevent nearly $50 \%$ of $B$. pertussis exposures annually, with an estimated cost savings of $\$ 2.38$ for each dollar invested in HCW vaccination. ${ }^{15}$ Further studies of the cost-effectiveness of the use of Tdap vaccines in HCWs are needed.
Tdap vaccines are additional "patient safety" vaccines that, like influenza vaccine, protect both HCWs and their patients. Given the unacceptably low rates of HCW influenza vaccination (a topic discussed in several articles in this issue of the journal) and the recent push to increase these rates, ${ }^{30}$ a similar strategy for enhancing Tdap vaccination of HCWs is also needed. The awareness among HCWs of the importance of nosocomial pertussis, of the overall increase in the burden of $B$. pertussis disease, of the atypical presentation of pertussis in adolescents and adults, and of the rationale for HCW vaccination with Tdap vaccines must be heightened. As with influenza vaccination, Tdap vaccination campaigns must be multifaceted, backed by the healthcare facility's administration, and adequately supported with financial and human resources. Hopefully, because there is no need for annual revaccination and because there are few concerns about adverse events associated with Tdap vaccines, healthcare facilities can achieve a more acceptable level of adherence to HCW pertussis vaccination than has been historically noted for influenza vaccination.

In summary, the reports in this issue about pertussis in healthcare settings and the difficulties of infection control and postexposure prophylaxis associated with these outbreaks are of great concern. However, the licensure of Tdap vaccines and the demonstration of their efficacy suggest that universal administration of Tdap vaccine to HCWs could markedly reduce, if not possibly eliminate, the challenging problem of healthcare-associated pertussis.

Address reprint requests to Thomas R. Talbot, MD, MPH, A-2200 Medical Center North, 116121 st Avenue South, Vanderbilt University Medical Center, Nashville, TN 37232 (tom.talbot@vanderbilt.edu).

K.M.E. has received support from GlaxoSmithKline, Merck, Medimmune, and VaxGen. T.R.T. has received research funding from Nabi Pharmaceuticals and support from GlaxoSmithKline.

\section{REFERENCES}

1. Broder KR, Cortese MM, Iskander JK, et al. Preventing tetanus, diphtheria, and pertussis among adolescents: use of tetanus toxoid, reduced diphtheria toxoid and acellular pertussis vaccines recommendations of the Advisory Committee on Immunization Practices (ACIP). MMWR Recomm Rep 2006; 55:1-34.

2. National, state, and urban area vaccination coverage among children aged 19-35 months-United States, 2003. MMWR Morb Mortal Wkly Rep 2004; 53:658-661.

3. Kurt TL, Yeager AS, Guenette S, Dunlop S. Spread of pertussis by hospital staff. JAMA 1972;221:264-267.

4. Outbreaks of pertussis associated with hospitals-Kentucky, Pennsylvania, and Oregon, 2003. MMWR Morb Mortal Wkly Rep 2005; 54:67-71.

5. Addiss DG, Davis IP, Meade BD, et al. A pertussis outbreak in a Wisconsin nursing home. J Infect Dis 1991; 164:704-710.

6. Bassinet L, Matrat M, Njamkepo E, Aberrane S, Housset B, Guiso N. Nosocomial pertussis outbreak among adult patients and healthcare workers. Infect Control Hosp Epidemiol 2004; 25:995-997.

7. Boulay BR, Murray CJ, Ptak J, Kirkland KB, Montero J, Talbot EA. An outbreak of pertussis in a hematology-oncology care unit: implications 
for adult vaccination policy. Infect Control Hosp Epidemiol 2006; 27: 92-95.

8. Gehanno JF, Pestel-Caron M, Nouvellon M, Caillard JF. Nosocomial pertussis in healthcare workers from a pediatric emergency unit in France. Infect Control Hosp Epidemiol 1999; 20:549-552.

9. Karino T, Osaki K, Nakano E, Okimoto N. A pertussis outbreak in a ward for the severely retarded [in Japanese]. Kansenshogaku Zasshi 2001; 75:916-922.

10. Steketee RW, Wassilak SG, Adkins WN Jr, et al. Evidence for a high attack rate and efficacy of erythromycin prophylaxis in a pertussis outbreak in a facility for the developmentally disabled. J Infect Dis 1988; 157:434-440.

11. Weber DJ, Rutala WA. Pertussis: an underappreciated risk for nosocomial outbreaks. Infect Control Hosp Epidemiol 1998; 19:825-828.

12. De Serres G, Shadmani R, Duval B, et al. Morbidity of pertussis in adolescents and adults. J Infect Dis 2000; 182:174-179.

13. Wright SW, Decker MD, Edwards KM. Incidence of pertussis infection in healthcare workers. Infect Control Hosp Epidemiol 1999; 20:120-123.

14. Ward A, Caro J, Bassinet L, Housset B, O'Brien JA, Guiso N. Health and economic consequences of an outbreak of pertussis among healthcare workers in a hospital in France. Infect Control Hosp Epidemiol 2005; 26:288-292.

15. Calugar A, Ortega-Sanchez IR, Tiwari T, Oakes L, Jahre JA, Murphy TV. Nosocomial pertussis: costs of an outbreak and benefits of vaccinating health care workers. Clin Infect Dis 2006; 42:981-988.

16. Bryant $\mathrm{K}$, Humbaugh $\mathrm{K}$, Brothers $\mathrm{K}$, et al. Measures to control an outbreak of pertussis in a neonatal intermediate care nursery after exposure to a healthcare worker. Infect Control Hosp Epidemiol 2006; 27:541-545 (in this issue).

17. Cherry JD. Epidemiology of pertussis. Pediatr Infect Dis $J$ 2006; 25: 361-362.

18. Senzilet LD, Halperin SA, Spika JS, Alagaratnam M, Morris A, Smith B. Pertussis is a frequent cause of prolonged cough illness in adults and adolescents. Clin Infect Dis 2001; 32:1691-1697.

19. Hewlett EL, Edwards KM. Clinical practice. Pertussis-not just for kids. $N$ Engl J Med 2005; 352:1215-1222.

20. Bamberger E, Starets-Haham O, Greenberg D, et al. Adult pertussis is hazardous for the newborn. Infect Control Hosp Epidemiol 2006; 27: 623-625 (in this issue).

21. Tiwari T, Murphy TV, Moran J. Recommended antimicrobial agents for the treatment and postexposure prophylaxis of pertussis: 2005 CDC Guidelines. MMWR Recomm Rep 2005; 54:1-16.

22. Giugliani C, Vidal-Trécan G, Traore S, et al. Feasibility of azithromycin prophylaxis during a pertussis outbreak among healthcare workers in a university hospital in Paris. Infect Control Hosp Epidemiol 2006; 27: 626-629 (in this issue).

23. Güriş D. Treatment and chemoprophylaxis [chapter 3, amended]. In: National Immunization Program, Centers for Disease Control and Prevention. Guidelines for the Control of Pertussis Outbreaks, 2000. Available at: http:/www.cdc.gov/nip/publications/pertussis/guide.htm. Accessed April 12, 2006.

24. American Academy of Pediatrics. 2003 Red Book Online. Available at: http://aapredbook.aappublications.org/. Accessed April 3, 2006.

25. Devasia RA, Jones TF, Collier B, Schaffner W. Compliance with erythromycin versus azithromycin during a pertussis outbreak-Tennessee, 2004. In: Program and abstracts of the 43rd Annual Meeting of the Infectious Diseases Society of America; October 6-9, 2005; San Francisco, CA. Abstract 340.

26. Advisory Committee on Immunization Practices (ACIP), Centers for Disease Control and Prevention. ACIP votes to recommend use of combined tetanus, diphtheria and pertussis (Tdap) vaccine for adults. March 2, 2006. Available at: http://www.cdc.gov/nip/vaccine/tdap/. Accessed on April 12, 2005.

27. Ward JI, Cherry JD, Chang SJ, et al. Efficacy of an acellular pertussis vaccine among adolescents and adults. $N$ Engl J Med 2005; 353: 1555-1563.

28. Campins-Marti M, Cheng HK, Forsyth $K$, et al. Recommendations are needed for adolescent and adult pertussis immunisation: rationale and strategies for consideration. Vaccine 2001; 20:641-646.

29. Plotkin S. The global pertussis initiative: process overview. Pediatr Infect Dis $J$ 2005; 24:S7-9.

30. Talbot TR, Bradley SE, Cosgrove SE, Ruef C, Siegel JD, Weber DJ. Influenza vaccination of healthcare workers and vaccine allocation for healthcare workers during vaccine shortages. Infect Control Hosp Epidemiol 2005; 26:882-890. 